\title{
A conserved function of the zinc finger transcription factor Sp8/9 in allometric appendage growth in the milkweed bug Oncopeltus fasciatus
}

\author{
Nina D. Schaeper • Nikola-Michael Prpic • \\ Ernst A. Wimmer
}

Received: 9 June 2009 / Accepted: 30 August 2009 / Published online: 16 September 2009

(C) The Author(s) 2009. This article is published with open access at Springerlink.com

\begin{abstract}
The genes encoding the closely related zinc finger transcription factors Buttonhead (Btd) and D-Sp1 are expressed in the developing limb primordia of Drosophila melanogaster and are required for normal growth of the legs. The $D$-Spl homolog of the red flour beetle Tribolium castaneum, Sp 8 (appropriately termed $S p 8 / 9$ ), is also required for the proper growth of the leg segments. Here we report on the isolation and functional study of the Sp8/9 gene from the milkweed bug Oncopeltus fasciatus. We show that $S p 8 / 9$ is expressed in the developing appendages throughout development and that the downregulation of $S p 8 / 9$ via RNAi leads to antennae, rostrum, and legs with shortened and fused segments. This supports a conserved role of $S p 8 / 9$ in allometric leg segment growth. However, all leg segments including the claws are present and the expression of the leg genes Distal-less, dachshund, and homothorax are proportionally normal, thus providing no evidence for a role of $S p 8 / 9$ in appendage specification.
\end{abstract}

Keywords Sp transcription factors · Leg development . Allometric organ growth - Appendage evolution . Insect development

Communicated by S. Roth

N. D. Schaeper • N.-M. Prpic • E. A. Wimmer $(\bowtie)$

Georg-August-Universität,

Johann-Friedrich-Blumenbach-Institut für Zoologie

und Anthropologie, Abteilung Entwicklungsbiologie, GZMB,

Ernst-Caspari-Haus, Justus-von-Liebig-Weg 11,

37077 Göttingen, Germany

e-mail: ewimmer@gwdg.de

\section{Introduction}

The members of the $S p$ gene family encode evolutionarily conserved proteins, which are characterized by the presence of three zinc finger motifs (for nucleic acid binding) as well as an additional short conserved motif called Btd box (a transactivation domain), and are involved in a variety of developmental processes in both insects and vertebrates (reviewed in Zhao and Meng 2005). In Drosophila, two different Sp factor encoding genes, buttonhead (btd) and $D-S p 1$, have been shown to be expressed during leg development (Wimmer et al. 1996; Schöck et al. 1999; Estella et al. 2003). The btd gene is required for the specification and formation of the entire leg disc primordia (Estella et al. 2003; McKay et al. 2009). The gene is acting upstream of the wellcharacterized leg specification gene Distal-less $(D l l)$ and seems to be capable of activating the entire leg developmental cascade when expressed ectopically (Estella et al. 2003). The role of the $D-S p 1$ gene during leg development is less clear, but seems to be partially redundant with btd (Schöck et al. 1999; Estella et al. 2003). The homolog of $D-S p 1$ in the red flour beetle Tribolium castaneum, $S p 8$, has been shown to be involved in the control of allometric growth of the leg segments, but the evidence for a role of $\operatorname{Sp} 8$ in the specification of the entire leg primordium is ambiguous (Beermann et al. 2004). Orthologous genes to $D-S p 1$ and Tc $S p 8$ have also been identified in the mouse, termed $S p 8$ and Sp9 (Bell et al. 2003; Treichel et al. 2003; Kawakami et al. 2004). Please note that the murine $\operatorname{Sp} 8$ gene has also been called inaccurately $\mathrm{mBtd}$ (Treichel et al. 2003), because it was named at a time when its orthology to either Drosophila btd or Drosophila D-Sp1 was unclear. Intriguingly, both murine genes are also involved in limb outgrowth.

Here we present the isolation of an Sp factor encoding gene from the milkweed bug $O$. fasciatus. Although our 
analysis of the Oncopeltus gene confidently places it as a homolog of the Drosophila D-Sp1, Tribolium Sp8, and the murine $S p 8$ and $S p 9$ genes, the exact orthology within this group is unclear and we therefore designate the Oncopeltus gene as $S p 8 / 9$. We have studied the role of the Oncopeltus Sp8/9 ortholog in order to investigate its evolutionary conservation in insect appendage development. We find that $S p 8 / 9$ is expressed in the appendages throughout development and the downregulation of $S p 8 / 9$ expression via RNAi leads to shortened legs, rostrum, and antennae. These data show that $S p 8 / 9$ is involved in the allometric growth of the appendages. However, we find no evidence for a more global role of $S p 8 / 9$ in appendage specification.

\section{Materials and methods}

Animal husbandry and embryology

Milkweed bugs were reared as described previously (Hughes and Kaufman 2000). Collected eggs were kept at $25^{\circ} \mathrm{C}$. Embryos of all stages were fixed as reported in Liu and Kaufman (2004a). Oncopeltus embryo dissections before in situ staining were performed under a fluorescence stereomicroscope using SYTOX Green nucleic acid stain (Invitrogen) (Liu and Kaufman 2004b).

\section{Isolation of $S p 8 / 9$ and sequence analysis}

Oncopeltus embryos from 0 to $96 \mathrm{~h}$ were used for mRNA isolation using the MicroPoly(A)Purist kit (Ambion). This mRNA served as template for double-stranded (ds) cDNA synthesis (SMART PCR cDNA Synthesis kit, Clontech) and RACE template synthesis (SMART RACE cDNA Amplification Kit, Clontech). PCR with the primer pair Fw_GGC MGG GCI ACI TGY GAY TGY CCI AAY TG (GRATCDCPNC) and Rev_ARR TGR TCI SWI CKC ATR AAI CKY AA (LHDSRMFRK) resulted in a fragment of $311 \mathrm{bp}$. PCR fragments were cloned into the pCRII vector (Invitrogen). Additional sequence information was obtained by $5^{\prime}$ RACE PCR using the reverse primer CAG GTG AGC CTT GAG GTG CGA GGT C. Phylogenetic analysis of different $\mathrm{Sp}$ factor sequences was performed as described previously (Prpic et al. 2005). The Oncopeltus Sp8/9 sequence is available from the EMBL nucleotide database under the accession number FN396612.

In situ hybridization

The longest $5^{\prime}$ RACE fragment of $\operatorname{Sp} 8 / 9$ (1,078 bp comprising 181 bp $5^{\prime}$ UTR and 897 bp ORF) served as template for the synthesis of digoxygenin-labeled RNA probes (Roche). In situ hybridization was performed as described previously (Liu and Kaufman 2004a).

\section{Parental RNA interference}

The template for dsRNA synthesis was prepared by PCR with T7 (GAA TTG TAA TAC GAC TCA CTA TAG G) and Sp6-T7 (TAA TAC GAC TCA CTA TAG GAT TTA GGT GAC ACT ATA GA) primers from the longest 5' RACE fragment of $S p 8 / 9$ that has also been used for probe generation. Double-stranded RNA (dsRNA) was generated using the MEGAscript T7 Kit (Ambion) and resuspended in $1 \times$ injection buffer $\left(1.4 \mathrm{mM} \mathrm{NaCl}, 0.07 \mathrm{mM} \mathrm{Na}_{2} \mathrm{HPO}_{4}\right.$, $0.03 \mathrm{mM} \mathrm{KH}_{2} \mathrm{PO}_{4}, 4 \mathrm{mM} \mathrm{KCl}$ ) at a concentration of $4 \mu \mathrm{g} / \mu \mathrm{l}$. RNA injections in adult virgin Oncopeltus females were performed as described previously (Liu and Kaufman 2004a). Injection of $1 \times$ injection buffer served as the negative control. To verify the RNAi phenotypes obtained with the full fragment, we repeated the parental RNAi with two shorter non-overlapping fragments of $O f S p 8 / 9$. A different 5' RACE Of Sp8/9 fragment of 1,057 bp (133 bp 5' UTR, 924 bp ORF) was cut with XhoI which resulted in two fragments of 421 and $636 \mathrm{bp}$. Parental RNAi experiments with dsRNA transcribed from these two fragments resulted in the same phenotype with a similar frequency as for dsRNA injections with the full longest $5^{\prime}$ RACE fragment (data not shown). As independent RNAi controls, we performed injections of dsRNA of EGFP and $O f$ eve which resulted in no abnormal phenotype or the same phenotypes as previously published for Of eve, respectively (Liu and Kaufman 2005) (data not shown).

\section{Results and discussion}

Isolation of the $S p 8 / 9$ homolog of $O$. fasciatus

A short fragment of the Oncopeltus homolog of the Sp8/9 gene was isolated by PCR. More sequence information was obtained by $5^{\prime}$ RACE PCR. The available sequence comprises $181 \mathrm{bp}$ of $5^{\prime}$ UTR followed by $1,008 \mathrm{bp}$ of protein coding sequence also encoding the Btd box motif and the zinc finger motifs ZF 1, ZF 2, and a portion of ZF 3. An alignment of these conserved domains with the nine Sp factors from the mouse, as well as D-Sp1 and Btd from Drosophila and Tc Sp8 and Tc Btd (Schinko et al. 2008) from Tribolium, shows that the Btd box motif and the available sequences of the zinc finger motifs are almost identical in all Sp8/9 proteins, but clearly different from the homologous motifs in other Sp proteins (Fig. 1a). The linker regions between these motifs are also highly conserved in all $\mathrm{Sp} 8 / 9$ proteins, whereas differences in sequence and linker length exist in other Sp proteins (Fig. 1a). 


\section{Btd box}

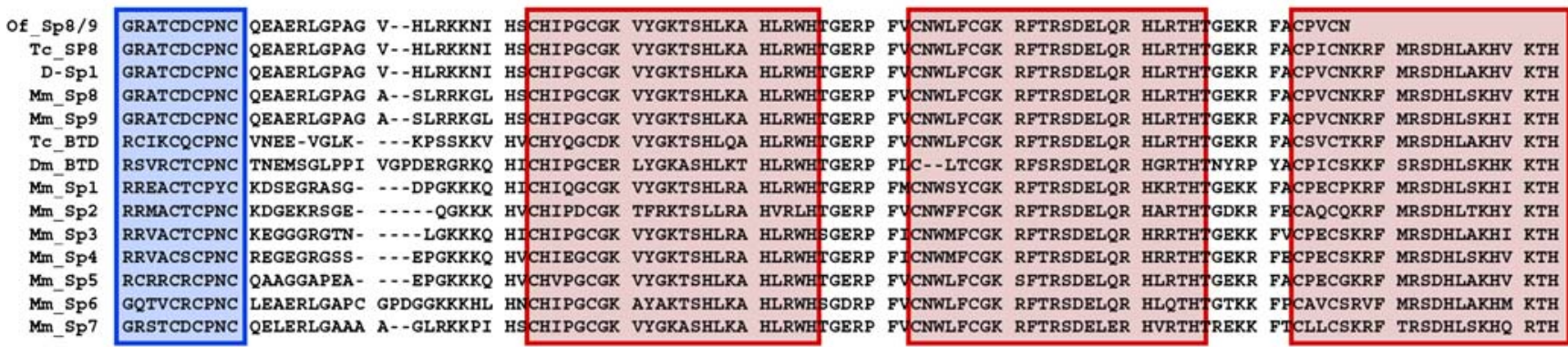

a

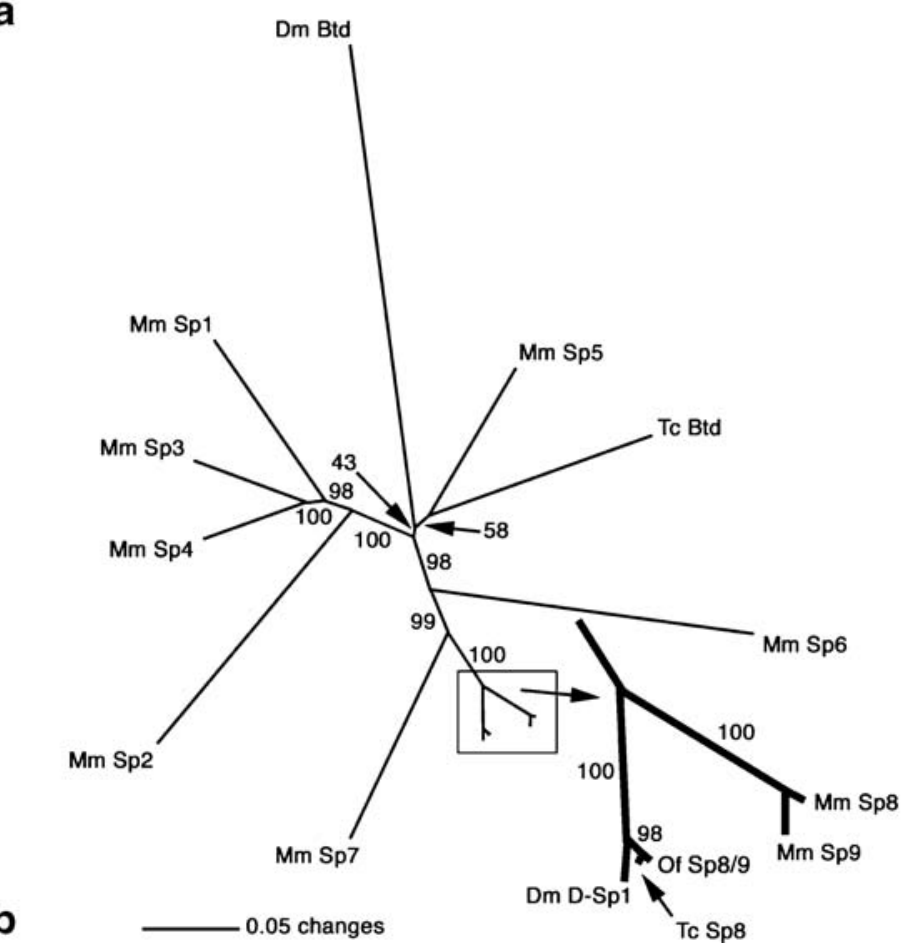

Fig. 1 Alignment and phylogenetic analysis of the Sp8/9 sequence from Oncopeltus. a Alignment of Sp proteins from Drosophila, Oncopeltus, Tribolium, and mouse. Shown is the protein region containing the Btd box motif (blue) and the three zinc finger motifs (red). The sequences of the $\mathrm{Sp} 8 / 9$ proteins including the D-Sp1 protein are almost identical and clearly different from other Sp proteins. b Phylogenetic analysis of all murine $\mathrm{Sp}$ factors and $\mathrm{Sp}$ factors from Tribolium, Drosophila, and Oncopeltus based on the alignment shown in (a). Shown is the unrooted majority rule consensus computed from 1,000 intermediate trees produced

In order to further corroborate the orthology of the $S p 8 / 9$ fragment isolated from Oncopeltus, we also performed a phylogenetic analysis (Fig. 1b) using the alignment in Fig. 1a in a maximum likelihood analysis with Tree Puzzle (Strimmer and von Haeseler 1996). Most edges in the phylogenetic tree are well supported with reliability values above 95. The Sp8/9 factors from Oncopeltus, Tribolium, and mouse cluster together in a group supported by the maximum reliability value of 100 and with very short edges, indicating that these genes are closely related. This grouping also includes D-Sp1 from Drosophila. These results further support the orthology of the Oncopeltus Sp8/9 with the quartet puzzling method (Strimmer and von Haeseler 1996). The numbers at the tree edges indicate the reliability values. GenBank accession numbers: Dm btd NP 511100, D-Spl NP 572579, Tc btd NP 001107792, Tc Spp NP 001034509, Mm Sppl NP 038700, $M m \_S p 2$ NP_084496, Mm_Sp3 NP_035580, Mm_Sp4 NP_033265, $M m \_S p 5$ NP_071880,Mm_Sp6 NP_112460, Mm_Sp7 NP_569725, $M m$ Sp8 NP $796056, M m$ Sp 9 NM 001005343. Species abbreviations: Mm Mus musculus, Tc Tribolium castaneum, Of Oncopeltus fasciatus, Dm Drosophila melanogaster

gene with the other $5 p 8 / 9$ genes and also give additional evidence to the previously published conclusion that the Drosophila D-Spl gene is actually the Drosophila Sp8/9 homolog (Beermann et al. 2004).

Sp8/9 is expressed during appendage development in $O$. fasciatus

Next we examined the expression profile of $S p 8 / 9$ in Oncopeltus embryos using whole mount in situ hybridization. During early stages of germband elongation, strong expression of $S p 8 / 9$ is seen in the limb buds of the thoracic 
legs and the antennae (Fig. 2a). Interestingly, the expression in the thoracic limb buds extends from there into the lateral tissue of the thoracic segments posterior to the limb buds, and thus is not restricted to the limb buds (Fig. 2a, arrows). A weaker expression is detected in the maxillary and labial segment, where distinct buds are not yet developed. A separate expression domain is present in the anterior head in the region where the labrum will develop. As the germband grows, a punctate expression in the ventral nervous system appears and the expression in the thorax becomes restricted to the leg limb buds (Fig. 2b). During later germband elongation stages, the punctate expression pattern in the ventral nervous system becomes stronger and some cells in the brain also express $S p 8 / 9$ (Fig. 2c). These brain cells are located at the rim of the head lobes in the area where the optic centers develop and also nearer to the ventral midline in the protocerebrum (Fig. 2c). All limb buds, including the emerging buds of the labrum, the mandibles, and the maxillae, now express $S p 8 / 9$. In fully elongated embryos, the expression pattern persists, but the
Fig. 2 Expression of $S p 8 / 9$ in Oncopeltus embryos. a Early germband elongation stage. Arrows point to expression extending from the thoracic limb buds into the lateral tissue of the thoracic segments posterior to the limb buds. b Mid-elongation germband stage. c Embryo approaching the final stages of germband elongation. d Fully elongated germband stage. e Retracted germband stage. f Magnification of a leg at the retracted germband stage showing the expression rings of $\mathrm{Sp} 8$ / 9 abutting the presumptive joint constrictions (arrowheads). All embryos are oriented with the anterior end to the left. Abbreviations: $l r$ labrum, an antenna, $m d$ mandible, $m x$ maxilla, $l b$ labium,

$t 1$ prothoracic leg,

t2 mesothoracic leg,

t3 metathoracic leg
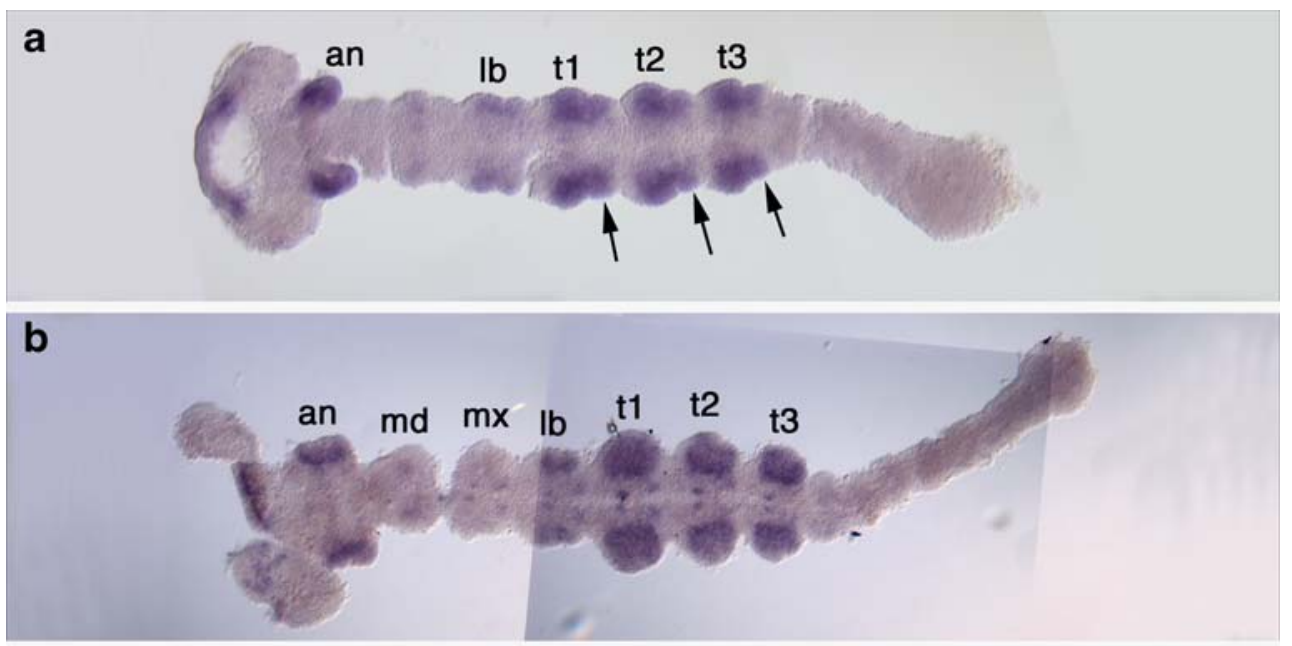

c
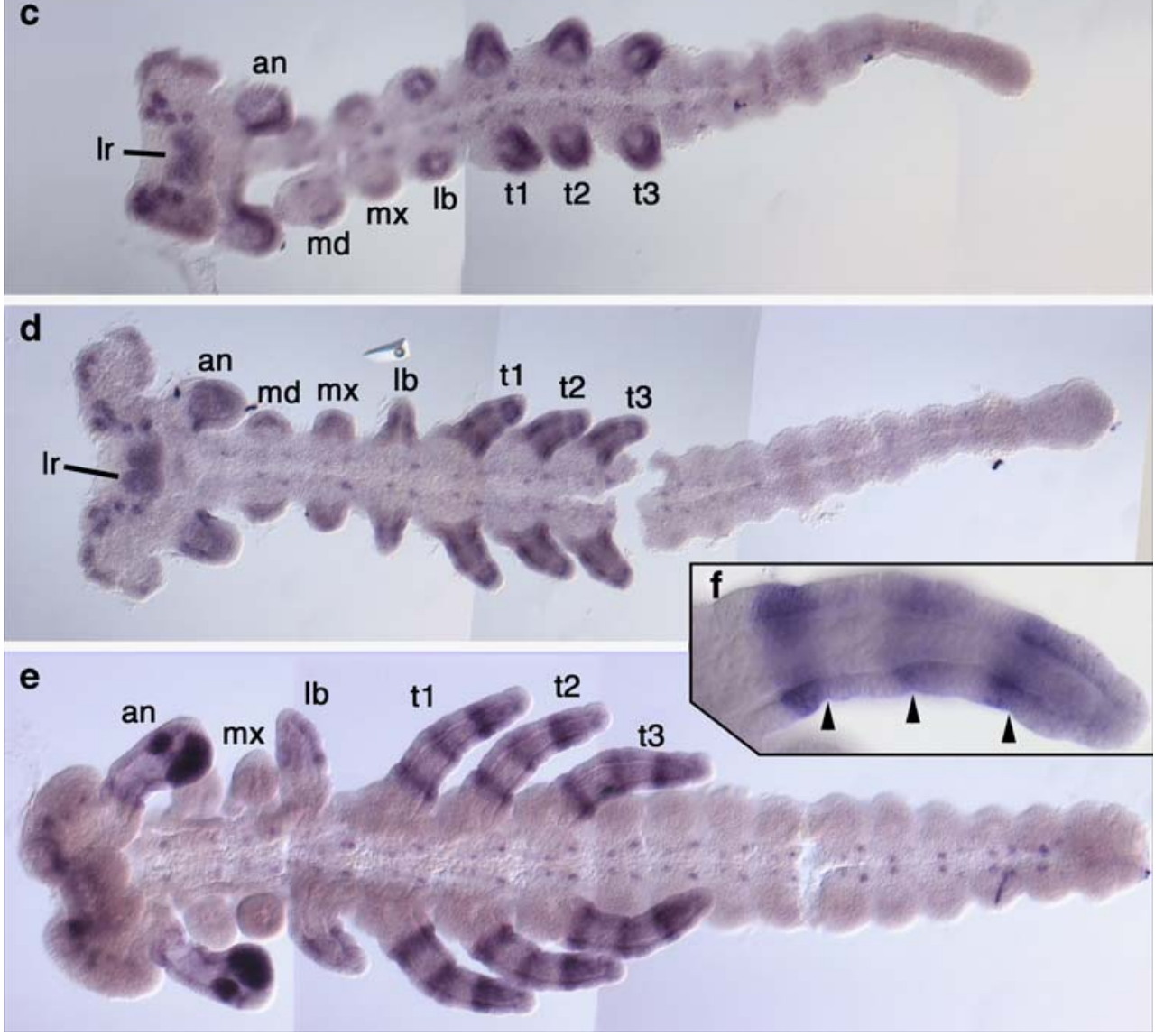
expression in the thoracic legs becomes slightly nonhomogeneous (Fig. 2d), indicating the emergence of iterated rings in the legs at later stages. At fully retracted germband stages, the expression pattern in the appendages is more restricted (Fig. 2e). In the antenna, $S p 8 / 9$ is restricted to a large ventral patch at the distal end and a smaller dot dorsal to it. The expression in the mandible has ceased and the expression in the maxilla is very weak. In the labium, Sp8/9 expression is largely confined to a faint ring near the middle of the appendage. In the thoracic legs, the expression is seen in three rings. These expression rings are located proximally adjacent to the constrictions that mark the presumptive joints between the leg segments (podomeres; note that not all future podomeres are yet developed at this stage of development) (Fig. 2f).

\section{Functional analysis of $S p 8 / 9$ using RNAi}

In order to establish the function of $S p 8 / 9$ during development of Oncopeltus, we have applied parental RNAi (Liu and Kaufman 2004a). The results of these injections are summarized in Table 1. In wild-type embryos, shortly before hatching, the appendages lie flat on the body and reach the posterior end of the abdomen (Fig. 3d, e). In Sp8/9 RNAi embryos, the appendages are severely shortened. They stick out (Fig. 3a) and do not reach further than the middle of the body (Fig. 3a, b).

In wild-type hatchlings the appendages are long and composed of several segments (podomeres) (Fig. 3f). The rostrum is a complex of four appendages (Fig. 3g). The labrum is thin and sharply pointed, the mandibles and the maxillae are long and thread-like, and the labium consists of four segments (Fig. 3g). The rostrum of the Sp8/9 RNAi animals is malformed (Fig. 3h). The labium is shortened; the distal segments are fused, bent, and enlarged at the tip. Therefore, the filiform mandibles and maxillae protrude from the labium at the distal end, while normally they are entirely ensheathed by it. The overall morphology of the mandibles, maxillae, and the labrum is normal in Sp8/9 RNAi animals, but they are shorter than in the wild type (Fig. 3h).

The wild-type antennae consist of a basal antennifer and four antennal segments (Fig. 3i). The antennae are severely affected in all Sp8/9 RNAi animals (Fig. 3c, j). The antennifer and the first segment of the antenna are roughly identical in size and shape to the wild type, but the three distal antennal segments are entirely fused and the antennal appendage as a whole is severely shortened. In about half of the cases, the fused distal antennal portion displays small ectopic outgrowths (arrow in Fig. 3j).

The wild-type thoracic legs consist of a short coxa, a trochanter which is closely attached to the femur, a tibia, and a two-segmented tarsus with two claws (Fig. 3k). The legs of Sp8/9 RNAi animals are much shorter than in the wild type (Fig. 3c, 1, m). All leg segments are present but some of them are fused together. The tarsal segments are always fused, but they can still be distinguished because constrictions indicating the rudimentary joints are still present. The trochanter and femur are also always fused. In some specimens, the proximal podomeres are so severely malformed and fused that they cannot be distinguished anymore (Fig. $3 \mathrm{~m}$ ). In contrast to these joints, the joints between femur and tibia and between tibia and tarsus are always present.

\section{Expression of Distal-less, dachshund, and homothorax in $S p 8 / 9$ RNAi embryos}

Since the results of the RNAi experiments indicated a role of $5 p 8 / 9$ in appendage development, we next studied the expression of leg developmental genes expressed at different positions along the proximal-distal leg axis.

The gene Distal-less $(D l l)$ is expressed in the distal portion of all appendages except for the mandible (Fig. 4a, b) (Angelini and Kaufman 2004). In the head appendages, Dll is expressed in the tip of the labrum, the entire distal portion of the antenna, and the tips of maxilla and labium (Fig. 4a). The thoracic legs show two separate domains of $D l l$ expression: a large distal domain ("sock") and a ring of expression proximal to it (Fig. 4b). The antennae are shorter in Sp8/9 RNAi embryos, but they express $D l l$ in their distal region (Fig. 4c). In addition, Dll is expressed in the distal tips of labrum, maxilla, and labium in Sp8/9 RNAi embryos (Fig. 4c). The thoracic legs are much shorter in Sp8/9 RNAi embryos than in the wild type, but they show a "ring and sock" pattern of Dll expression similar to the wild-type legs (Fig. 4d).

The dachshund (dac) gene is in the wild-type expressed in a medial ring in the thoracic legs, and in

Table 1 Parental RNAi with $S p 8 / 9$

\begin{tabular}{|c|c|c|c|c|}
\hline & $\mathrm{WT} n(\%)$ & Unspecific phenotype $n(\%)$ & Appendage phenotype $n(\%)$ & Total $n$ \\
\hline Sp $8 / 9$ pRNAi & $4(2.4 \%)$ & $6(3.6 \%)$ & $160(94 \%)$ & 170 \\
\hline Control (injection buffer) & $104(95 \%)$ & $5(5 \%)$ & $0(0 \%)$ & 109 \\
\hline
\end{tabular}

Given are the total numbers $(n)$ of specimens in each category. WT stands for wild-type specimens after injection. Unspecific phenotypes were specimens that showed some development, but had died before any discernible morphology was formed. These phenotypes had a similar frequency in the $S p 8 / 9$ pRNAi and in the control injections. The appendage phenotype was obtained only in the Sp8/9 pRNAi 

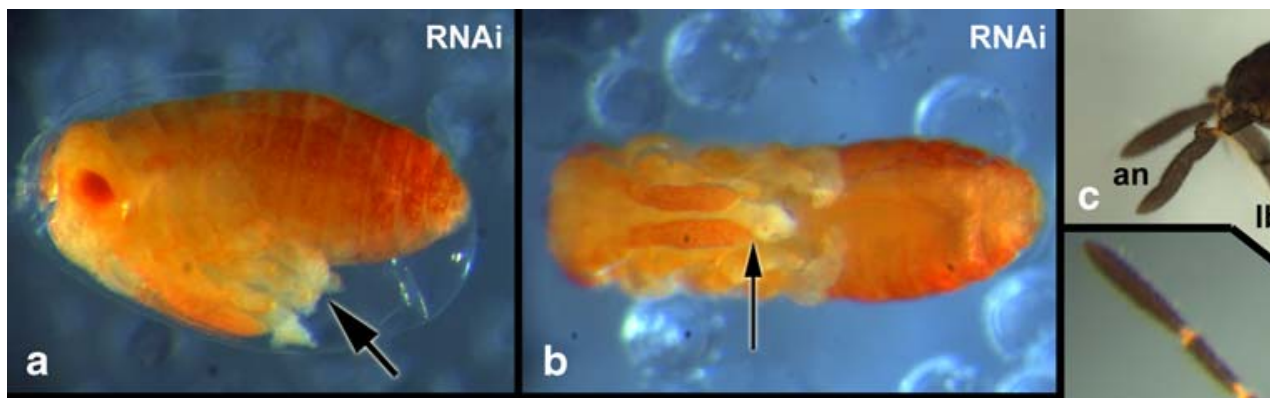

RNAi
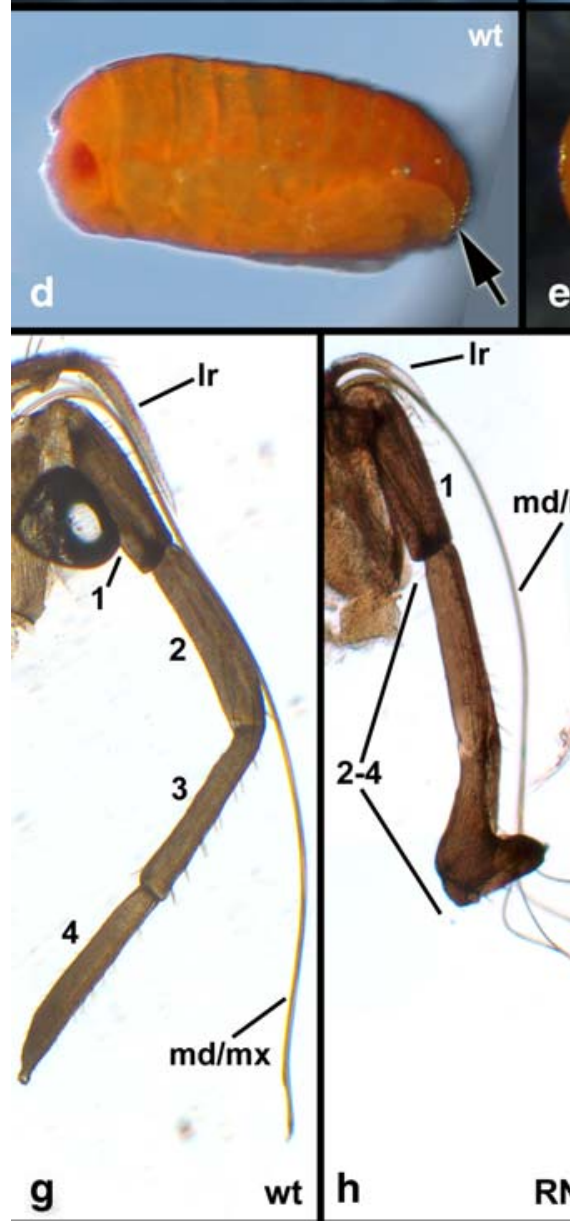

e

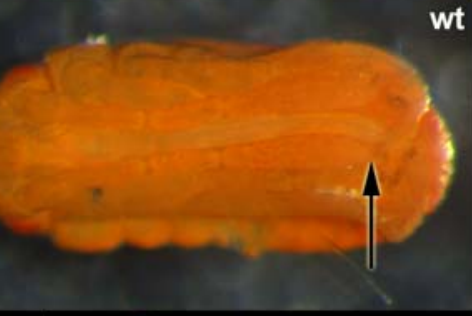

\section{t}
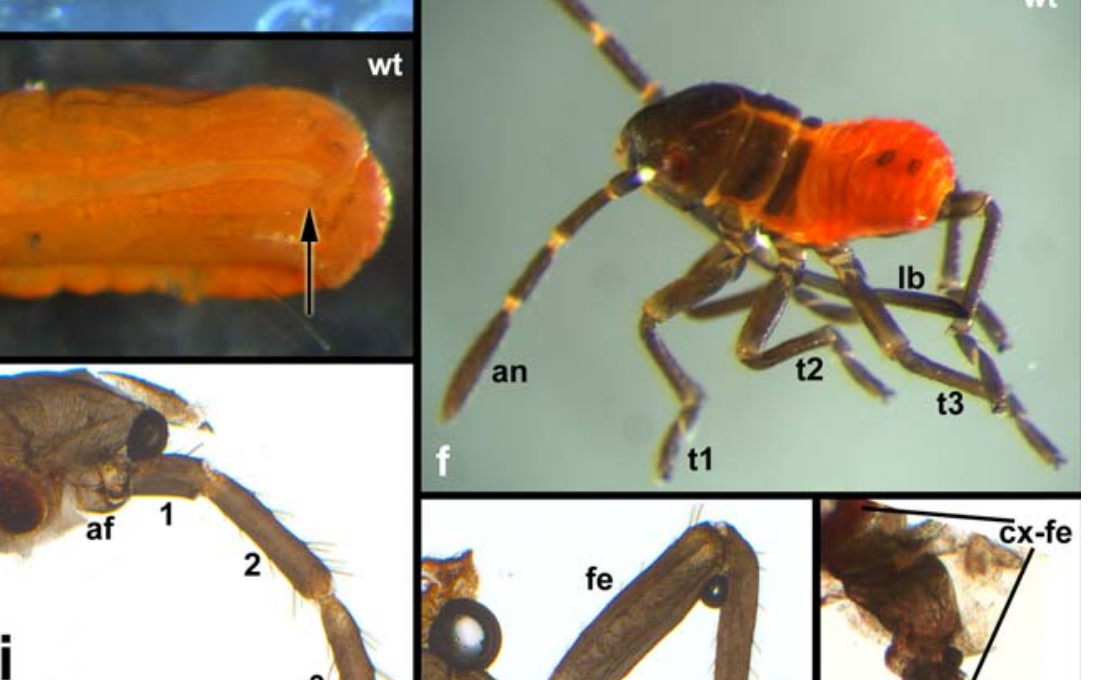

Fig. 3 Parental RNAi with Sp8/9 in Oncopeltus. a, b Embryos after Sp8/9 RNAi shortly before hatching in lateral (a) and ventral (b) aspect. c Hatchling after Sp8/9 RNAi. d, e Wild-type embryos shortly before hatching in lateral (d) and ventral (e) aspect. f Wild-type hatchling. The arrows in (a) and (d) denote the tips of the legs. The arrows in (b) and (e) denote the tips of the antennae. All animals in (a)-(f) are oriented with the anterior end to the left. $\mathbf{g}-\mathbf{m}$ Preparations of the appendages of wild-type hatchlings $(\mathbf{g}, \mathbf{i}, \mathbf{k})$ and hatchlings after Sp8/9 RNAi (h, j, l, m). The wild-type rostrum (g) comprises the foursegmented labium, filiform maxillae and mandibles, and the pointed labrum. The rostrum of RNAi animals (h) is malformed with fused distal segments. The wild-type antenna (i) comprises an antennifer and

four additional segments. The antenna of RNAi animals is severely shortened with fused segments (j). The arrow in (j) points to a small ectopic outgrowth on the antenna seen in about half of the hatchlings after Sp8/9 RNAi. The wild-type legs (k) comprise a coxa, trochanter, femur, tibia, and a two-segmented tarsus with two claws. The legs of RNAi animals are shortened with fused tarsal segments $(\mathbf{l}, \mathbf{m})$. The more proximal segments coxa, trochanter, and femur may be partially (I) or fully fused together (m). Abbreviations: $l r$ labrum, an antenna, af antennifer, ro rostrum, $m d$ mandible, $m x$ maxilla, $l b$ labium, $t 1$ prothoracic leg, $t 2$ mesothoracic leg, $t 3$ metathoracic leg, $c x$ coxa, $t r$ trochanter, $f e$ femur, $t i$ tibia, $t a$ tarsus, $c l$ claw

a thin ring near the base in the labium and in the antenna (Fig. 4e) (Angelini and Kaufman 2004). The dac gene is also expressed very strongly in the mandible and in the maxilla (Fig. 4e). In the Sp8/9 RNAi animals, this pattern is not significantly altered. The expression ring in the antenna is thinner, but the expression in the mandible, maxilla, and labium is unchanged (Fig. 4f, g). The thoracic legs are significantly shortened, but the medial ring 
Fig. 4 Expression of Distal-less, dachshund, and homothorax in Sp8/9 RNAi embryos. a, b Wildtype $D l l$ expression in the head region (a) and the thoracic legs (b). c, d Expression of $\mathrm{Dll}$ in Sp8/9 RNAi embryos. Please note that the brownish staining in the head and the bases of all appendages (including the mandibles) in (c, $\mathbf{d})$ is unspecific background sometimes produced during the staining procedure. Embryos in (a)-(d) are at the retracted germband stage. Note the expression in the tips of the maxilla and labium (arrowhead and arrow, respectively, in (a) and (c)). Also note the expression in a distal "sock" and a proximal "ring" in the thoracic legs (arrow and arrowhead, respectively, in (b) and (d)). e-g Expression of dac in wild-type (e) and Sp8/9 RNAi embryos (f, g). Please note that the embryo in (f) has been damaged during preparation (the head has come off and the t1 leg is damaged). The embryos in (e) and (f) are at the retracted germband stage and the embryo in (g) is at an even later retracted germband stage. $\mathbf{h}-\mathbf{i}$ Expression of $h$ th in wild-type (h) and Sp8/9 RNAi embryos (i). Both embryos are at the retracted germband stage. All animals are oriented with the anterior end to the left. Abbreviations: $h l$ head lobe, Ir labrum, an antenna, $m d$ mandible, $m x$ maxilla, $l b$ labium, $t 1$ prothoracic leg, $t 2$ mesothoracic leg, $t 3$ metathoracic leg, $p l$ pleuropodium
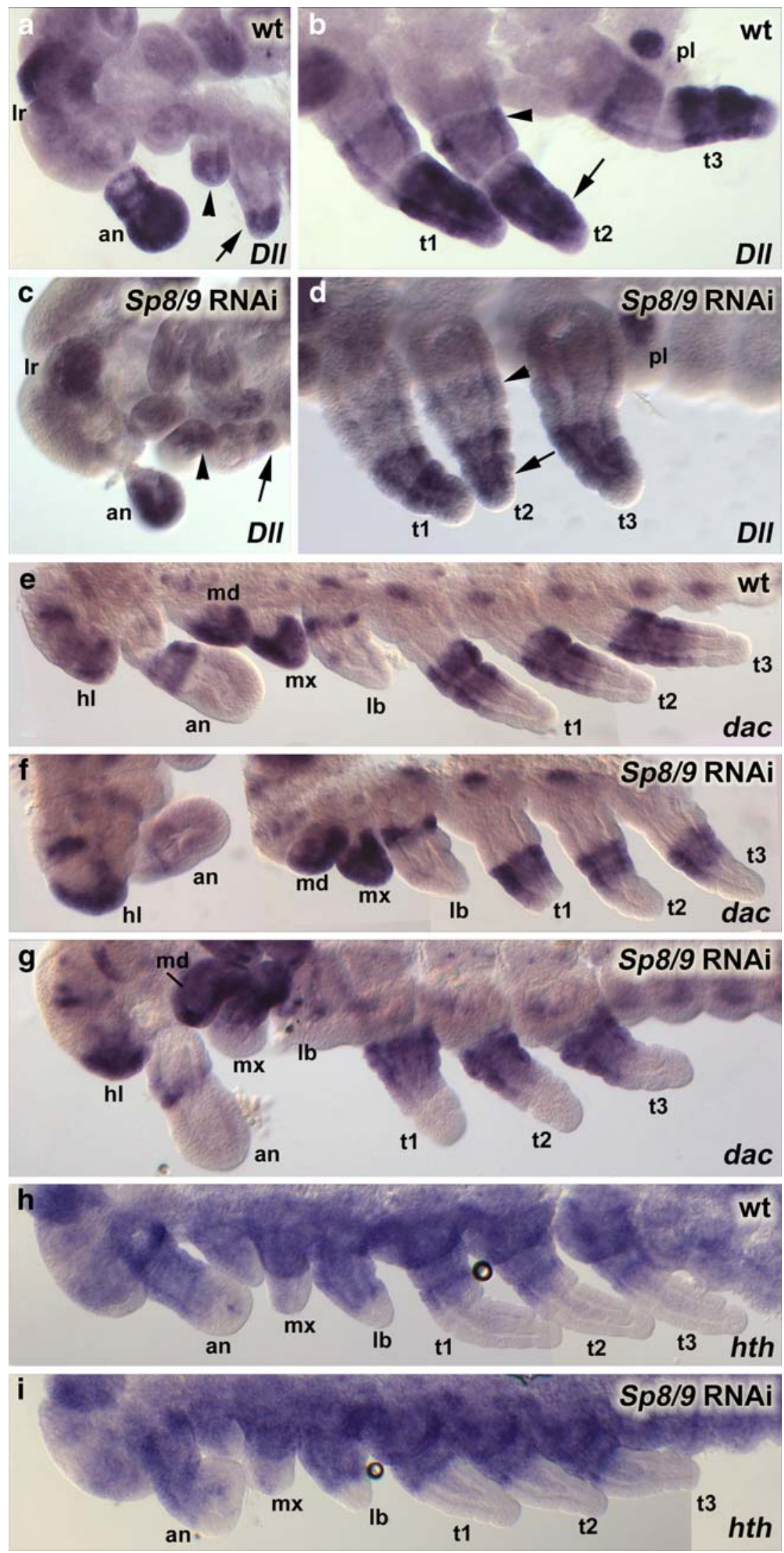
of $d a c$ expression is present as in the wild-type legs (Fig. 4f, g).

The gene homothorax (hth) is in the wild type expressed in the proximal area of all appendages (Fig. 4h), but the distal extension of expression is different in the different appendage types (Angelini and Kaufman 2004). Gene expression is restricted to the proximal third of the legs, but is expressed in the proximal two thirds of the appendage in antennae, mandible, and labium and fills the proximal half of the maxilla (Fig. 4h). This proportional pattern is identical in Sp8/9 RNAi animals although the legs are shorter than in the wild type (Fig. 4i).

A conserved role in appendage axis elongation

Previous work in Drosophila has shown that the D-Spl gene is expressed in the thoracic limb primordia in the embryo and in the leg imaginal discs in the larva (Wimmer et al. 1996; Schöck et al. 1999; Estella et al. 2003). In the leg discs, $D-S p 1$ is expressed in concentric rings that roughly correspond to the position of the future joints between the leg segments. This expression pattern is very similar to the late expression of $S p 8 / 9$ in the legs of the beetle $T$. castaneum. In this insect species, $S p 8 / 9$ is expressed in up to four segmental rings that lie at a similar location in the legs as the expression rings of the Serrate (Ser) gene, which encodes the ligand of the Notch $(\mathrm{N})$ receptor (Beermann et al. 2004). RNAi experiments with Sp8/9 have shown that the podomeres are severely shortened and sometimes fused, and that the number of Ser rings is also reduced. Based on these data, Beermann et al. (2004) suggested that Sp8/9 is involved in the control of allometric growth of the individual leg segments, probably by interfering with the Notch pathway, which is known to control allometric podomere growth in Drosophila and other arthropod species (de Celis et al. 1998; Rauskolb and Irvine 1999; Bishop et al. 1999; Prpic and Damen 2009). It has been noted previously that the process of leg segment growth is tightly linked with the process of joint formation, because both processes are regulated by the Notch pathway and its targets (Milán and Cohen 2000), and this would then also explain the observed podomere fusions.

Our results in Oncopeltus support an evolutionarily conserved role of $S p 8 / 9$ in the control of allometric podomere growth. First, the Sp8/9 expression pattern in the legs after full germband elongation is very similar to the pattern in Tribolium and consists of several rings adjacent to the constrictions of the future leg joints. Second, after RNAi, all leg segments are still present but severely shortened compared to the wild-type legs. In addition, the podomere fusions observed in the legs and in the antennae are compatible with the notion that the Notch pathway is also affected, because similar podomere fusion phenotypes are observed in Drosophila when members of effectors of the Notch pathway are impaired (e.g., de Celis et al. 1998; Rauskolb and Irvine 1999; Bishop et al. 1999).

No evidence for a role of $S p 8 / 9$ in appendage specification

There is some evidence in Drosophila that $D-S p 1$ has a role in leg specification by activating $\mathrm{Dll}$ expression in the embryonic leg primordia together with $\mathrm{Wg}$ and $\mathrm{Dpp}$ signaling and Dll autoregulation (Estella et al. 2003; McKay et al. 2009). However, this evidence is not conclusive because the experiments were not able to discriminate between the effect of $D-S p 1$ and the effect of the neighboring gene $b t d$. Beermann et al. (2004) suggest that $D l l$ is a target gene of Sp8/9 in Tribolium, although Dll is still expressed in the legs after Sp8/9 RNAi. In summary, the evidence for a role of $S p 8 / 9$ in leg specification and $D l l$ activation in the insects studied so far is inconclusive.

Our results with $S p 8 / 9$ RNAi in Oncopeltus provide no evidence for a role of $S p 8 / 9$ in appendage specification or Dll activation. All appendages are present in Sp8/9 RNAi animals and the legs consist of all podomeres including the distal claws. This suggests that the specification of the appendages and their overall proximal-distal patterning is not disrupted. This is further evidenced by the expression of the proximal-distal marker genes $\mathrm{Dll}$, dac, and hth in Sp8/9 RNAi animals. Although the legs in these animals are much shorter than in the wild type, the expression of Dll, dac, and $h t h$ is proportionally identical to the wild-type expression, indicating that proximal, medial, and distal fates are present. These data also suggest that $D l l, d a c$, and hth expression is not dependent on activation by $S p 8 / 9$. We note, however, that we found very weak residual expression of Sp8/9 after Sp8/9 RNAi (data not shown), and thus we cannot exclude the possibility that the phenotypes we obtained do not represent the loss-of-function (null) phenotype.

Acknowledgments NDS would like to thank Paul Z. Liu for introducing her to Oncopeltus work and Nipam H. Patel for support and advice. This work has been supported by an Education Abroad Program Grant and a Fulbright Travel Grant (both to NDS), the European Community's Marie Curie Research Training Network ZOONET under contract MRTN-CT-2004-005624 (to EAW) and by a DFG Emmy Noether Program grant PR 1109/1-1 (to NMP).

Open Access This article is distributed under the terms of the Creative Commons Attribution Noncommercial License which permits any noncommercial use, distribution, and reproduction in any medium, provided the original author(s) and source are credited.

\section{References}

Angelini DR, Kaufman TC (2004) Functional analyses in the hemipteran Oncopeltus fasciatus reveal conserved and derived aspects of appendage patterning in insects. Dev Biol 271:306-321 
Beermann A, Aranda M, Schröder R (2004) The Sp8 zinc-finger transcription factor is involved in allometric growth of the limbs in the beetle Tribolium castaneum. Development 131:733-742

Bell SM, Schreiner CM, Waclaw RR, Campbell K, Potter SS, Scott WJ (2003) Sp8 is crucial for limb outgrowth and neuropore closure. PNAS 100:12195-12200

Bishop SA, Klein T, Martinez Arias A, Couso JP (1999) Composite signalling from Serrate and Delta establishes leg segments in Drosophila through Notch. Development 126:2993-3003

de Celis JF, Tyler DM, de Celis J, Bray SJ (1998) Notch signalling mediates segmentation of the Drosophila leg. Development 125:4617-4626

Estella C, Rieckhof G, Calleja M, Morata G (2003) The role of buttonhead and Spl in the development of the ventral imaginal discs of Drosophila. Development 130:5929-5941

Hughes CL, Kaufman TC (2000) RNAi analysis of Deformed, proboscipedia and Sex combs reduced in the milkweed bug Oncopeltus fasciatus: novel Hox genes in the Hemipteran head. Development 127:3683-3694

Liu PZ, Kaufman TC (2004a) hunchback is required for suppression of abdominal identity, and for proper germband growth and segmentation in the intermediate germband insect Oncopeltus fasciatus. Development 131:1515-1527

Liu PZ, Kaufman TC (2004b) Kruppel is a gap gene in the intermediate germband insect Oncopeltus fasciatus and is required for development of both blastoderm and germbandderived segments. Development 131:4567-4579

Liu PZ, Kaufman TC (2005) even-skipped is not a pair-rule gene but has segmental and gap-like functions in Oncopeltus fasciatus, an intermediate germband insect. Development 132:2081-2092

Kawakami Y, Esteban CR, Matsui T, Rodriguez-Leon J, Kato S, Izpisua Belmonte JC (2004) Sp 8 and Sp9, two closely related buttonheadlike transcription factors, regulate $F g f 8$ expression and limb outgrowth in vertebrate embryos. Development 131:4763-4774
McKay DJ, Estella C, Mann RS (2009) The origins of the Drosophila leg revealed by the cis-regulatory architecture of the Distalless gene. Development 136:61-71

Milán M, Cohen SM (2000) Subdividing cell populations in the developing limbs of Drosophila: do wing veins and leg segments define units of growth control? Dev Biol 217:1-9

Prpic NM, Janssen R, Damen WGM, Tautz D (2005) Evolution of dorsal-ventral axis formation in arthropod appendages: H15 and optomotor-blind/bifid-type T-box genes in the millipede Glomeris marginata (Myriapoda: Diplopoda). Evol Dev 7:51-57

Prpic NM, Damen WGM (2009) Notch-mediated segmentation of the appendages is a molecular phylotypic trait of the arthropods. Dev Biol 326:262-271

Rauskolb C, Irvine KD (1999) Notch-mediated segmentation and growth control of the Drosophila leg. Dev Biol 210:339-350

Schinko JB, Kreuzer N, Offen N, Posnien N, Wimmer EA, Bucher G (2008) Divergent functions of orthodenticle, empty spiracles and buttonhead in early head patterning of the beetle Tribolium castaneum (Coleoptera). Dev Biol 317:600-613

Schöck F, Purnell BA, Wimmer EA, Jäckle H (1999) Common and diverged functions of the Drosophila gene pair $D-S p 1$ and buttonhead. Mech Dev 89:125-132

Strimmer K, von Haeseler A (1996) Quartet puzzling: a quartet maximum likelihood method for reconstructing tree topologies. Mol Biol Evol 13:964-969

Treichel D, Schöck F, Jäckle H, Gruss P, Mansouri A (2003) mBtd is required to maintain signalling during murine limb development. Genes Dev 17:2630-2635

Wimmer EA, Frommer G, Purnell BA, Jäckle H (1996) buttonhead and D-Sp1: a novel Drosophila gene pair. Mech Dev 59:53-62

Zhao C, Meng A (2005) Sp1-like transcription factors are regulators of embryonic development in vertebrates. Develop Growth Differ 47:201-211 